Draft version July 30, 2021

Typeset using $\mathrm{LAT}_{\mathrm{E}} \mathrm{X}$ twocolumn style in AASTeX61

\title{
STRUCTURAL AND PHOTOMETRIC PROPERTIES OF THE ANDROMEDA SATELLITE DWARF GALAXY LACERTA I FROM DEEP IMAGING WITH WIYN PODI
}

\author{
Katherine L. Rhode, ${ }^{1}$ Denija Crnojević, ${ }^{2}$ David J. Sand, ${ }^{2}$ Steven Janowiecki,${ }^{3}$ Michael D. Young, ${ }^{1}$ And \\ KRISTINE SPEKKENS ${ }^{4}$ \\ ${ }^{1}$ Indiana University Department of Astronomy, 727 East 3rd Street, Swain West 319, Bloomington, IN 47405, USA \\ ${ }^{2}$ Texas Tech University Department of Physics, Box 41051, Lubbock, TX 79409-1051, USA \\ ${ }^{3}$ International Centre for Radio Astronomy Research (ICRAR), University of Western Australia, 35 Stirling Highway, Crawley, WA 6009, \\ Australia \\ ${ }^{4}$ Department of Physics, Royal Military College of Canada, P.O. Box 17000, Station Forces, Kingston, Ontario, K7K 7B4, Canada
}

\section{ABSTRACT}

We present results from WIYN pODI imaging of Lacerta I (And XXXI), a satellite dwarf galaxy discovered in the outskirts of the Andromeda galaxy (M31) in Pan-STARRS1 survey data. Our deep, wide-field $g, i$ photometry reaches $\sim 3$ magnitudes fainter than the photometry in the Pan-STARRS1 discovery paper and allows us to trace the stellar population of Lac I beyond two half-light radii from the galaxy center. We measure a Tip of the Red Giant Branch (TRGB) distance for Lac I of $(m-M)_{0}=24.44 \pm 0.11 \mathrm{mag}(773 \pm 40 \mathrm{kpc}$, or $264 \pm 6 \mathrm{kpc}$ from M31), which is consistent with the Pan-STARRS1 distance. We use a maximum-likelihood technique to derive structural properties for the galaxy, and find a half-light radius $\left(r_{h}\right)$ of $3.24 \pm 0.21 \operatorname{arcmin}(728 \pm 47 \mathrm{pc})$, ellipticity $(\epsilon)$ of $0.44 \pm 0.03$, total magnitude $M_{V}=-11.4 \pm 0.3$, and central surface brightness $\mu_{V, 0}=24.8 \pm 0.3 \mathrm{mag} \operatorname{arcsec}^{-2}$. We find no HI emission in archival data and set a limit on Lac I's neutral gas mass-to-light ratio of $M_{H I} / L_{V}<0.06 M_{\odot} / L_{\odot}$, confirming Lac I as a gas-poor dwarf spheroidal galaxy. Photometric metallicities derived from Red Giant Branch stars within $2 r_{h}$ yield a median $[\mathrm{Fe} / \mathrm{H}]$ of $-1.68 \pm 0.03$, which is more metal-rich than the spectroscopically-derived value from Martin et al. (2014). Combining our measured magnitude with this higher metallicity estimate places Lac I closer to its expected position on the luminosity-metallicity relation for dwarf galaxies.

Keywords: Local Group - galaxies: individual (Lac I) - galaxies: individual (And XXXI) - galaxies: dwarf - galaxies: photometry

krhode@indiana.edu

denija.crnojevic@ttu.edu

david.sand@ttu.edu

steven.janowiecki@uwa.edu.au

youngmd@indiana.edu

Kristine.Spekkens@rmc.ca 


\section{INTRODUCTION}

The number, spatial distribution, mass function, and kinematics of the dwarf galaxies in an environment like that of the Local Group provide important tests for theoretical ideas about cosmology, dark matter, and galaxy formation (e.g., Klypin et al. 1999, Moore et al. 1999, Bullock \& Johnston 2005, Simon \& Geha 2007). Furthermore, dwarf galaxies themselves serve as valuable laboratories for our understanding of the physical processes involved in galaxy evolution, star formation, feedback, and chemical evolution (e.g., Mateo 1998, Tolstoy et al. 2009. McConnachie 2012 and references therein). The importance of dwarf galaxies to all of these areas of extragalactic astrophysics and cosmology has motivated an array of surveys and searches for additional low-mass galaxies in and around the Local Group (e.g., Willman et al. 2005; Martin et al. 2006; Belokurov et al. 2007; Bell et al. 2011; McConnachie et al. 2009; Adams et al. 2013; Bechtol et al. 2015; Koposov et al. 2015; Kim et al. 2015, Janesh et al. 2015).

In particular, the regions around the Andromeda Galaxy (M31) have lately been the focus of a number of dedicated searches for satellite dwarf galaxies, and have met with much success. For example, searches for stellar overdensities in photometric observations from the Sloan Digital Sky Survey (SDSS) and the Pan-Andromeda Archaeological Survey (PAndAS) have resulted in the discovery of tens of dwarf galaxies in regions around Andromeda (e.g., Zucker 2004, 2007; McConnachie et al. 2008; Martin et al. 2006, 2009; Bell et al. 2011; Slater et al. 2011). A recent contribution in this area comes from the Panoramic Survey Telescope and Rapid Response System 1 (Pan-STARRS1; Kaiser et al. 2010) $3 \pi$ survey. By searching through Pan-STARRS1 photometric source catalogs, Martin et al. (2013a) discovered two new dwarf galaxies, Lacerta I (And XXXI) and Cassiopeia III (And XXXII), in regions around Andromeda that had not been included in other systematic imaging surveys. In a follow-up paper, Martin et al. (2013b) described the discovery in Pan-STARRS1 imaging data of a third Andromeda satellite galaxy, Perseus I (And XXXIII), located in a region with shallow SDSS coverage. All three galaxies were confirmed to be satellite galaxies via a spectroscopic study by Martin et al.

(2014), who derived systemic radial velocities for each system as well as individual metallicity measurements for member stars (see Section 4.4 for more discussion). All three are located more than 10 degrees away from M31 in projection and have relatively faint central surface brightnesses $\left(\mu_{0} \approx 25-26\right.$ mag $\left.\operatorname{arcsec}^{-2}\right)$, properties which likely contributed to their late discovery (Martin et al. 2013a, 2013b). Cas III is located within the area of the thin rotating plane of M31 satellite galaxies (Ibata et al. 2013) but Martin et al. (2014) showed that it is moving in the opposite sense relative to the disk; Per I and Lac I are located on the far eastern and far western sides of Andromeda, respectively (Martin et al. 2013a,b).

Prompted in part by the installation and commissioning of a new camera on the WIYN 3.5-m telescope ${ }^{1}$ during the 2012-2013 observing season, we began a campaign to obtain deep, wide-field imaging of these and other selected nearby dwarf galaxies. The aim is to take advantage of the excellent image quality and depth made possible by WIYN to study the galaxies' structure and stellar populations out to large galactocentric radius. The first dwarf galaxy we targeted is Lac I, a relatively luminous dwarf $\left(M_{V} \sim-12\right)$ that lies $\sim 20^{\circ}(\sim 275 \mathrm{kpc})$ away from Andromeda in projected distance (Martin et al. 2013a). The photometry of Lac I presented in Martin et al. (2013a) reached $i \sim 22.5$ and yielded estimates of the distance, size, and metallicity of the galaxy. The Martin et al. (2014) spectroscopy study presented the systemic velocity and velocity dispersion $\left(v_{r, \text { helio }}=-198.4 \pm 1.4 \mathrm{~km} \mathrm{~s}^{-1}, \sigma_{v, r}=10.3 \pm 0.9 \mathrm{~km} \mathrm{~s}^{-1}\right)$ as well as a refined metallicity estimate and $V$-band massto-light ratio. In this paper, we present results from imaging of Lac I that reaches $\sim 24-25$ in the $g$ and $i$ filters, covers a $\sim 20^{\prime} \times 20^{\prime}$ area, and allows us to trace the stellar population beyond two half-light radii.

The paper is organized as follows. Section 2 describes the observations and initial data reduction and Section 3 discusses our methods for source detection, photometry, and completeness testing. Section 4 presents the properties we measure for Lac I, including the colormagnitude diagram (CMD), distance via the Tip of the Red Giant Branch (TRGB) method, structural parameters, luminosity, limits on the neutral gas content, and the photometrically-derived metallicity distribution function. The last section of the paper gives a summary and our final conclusions.

\section{OBSERVATIONS AND DATA REDUCTION}

Observations of Lac I were obtained on 2013 October 1 with the WIYN 3.5-m telescope and the One Degree Imager with a partially-filled focal plane (pODI; Harbeck et al. 2014). The pODI camera was comprised of nine orthogonal transfer arrays (OTAs) arranged in a 3x3 configuration, as well as four additional OTAs positioned at various radial locations around the focal

\footnotetext{
1 The WIYN Observatory is a joint facility of the University of Wisconsin-Madison, Indiana University, the National Optical Astronomy Observatory and the University of Missouri.
} 
plane. Each individual OTA is an $8 \times 8$ arrangement of orthogonal transfer CCD detectors with 480x496 12- $\mu$ m pixels. The central $3 \times 3$ array of OTAs in pODI, which provided a field-of-view of $\sim 24^{\prime} \times 24^{\prime}$ and a pixel scale of $0.11^{\prime \prime}$ pixel $^{-1}$, was used to image the target objects and the outlying OTAs were used for guiding during the exposure. (Note that pODI was upgraded in 2015 and is now referred to as the ODI camera; new detectors were added to create a $5 \times 6$ OTA configuration that provides a $40^{\prime} \times 48^{\prime}$ field-of-view.) We obtained nine 700-second exposures of the Lac I field in $g$ and another nine 600-second exposures in $i$ with pODI. The telescope was dithered between exposures in order to help eliminate gaps between the CCDs and OTAs during the subsequent image stacking process.

The pODI images were immediately transferred from WIYN to the ODI Pipeline, Portal, and Archive (ODIPPA; Gopu et al. 2014) $)^{2}$ at Indiana University and later processed with the QuickReduce pipeline (Kotulla 2014). The pipeline corrects each image for cross-talk, subtracts the overscan signal, corrects the images for non-linearity and persistence, applies bias, dark, flatfield, and pupil ghost corrections, and removes cosmic rays. The pipeline-processed images of Lac I were then flattened with a night-sky flat, reprojected to a common pixel scale, and finally average-combined to create a single deep, stacked image in each filter. Areas on the edges of the stacked images with slightly higher noise levels (because of reduced exposure time due to the dither pattern) were clipped to produce a usable field $\sim 20^{\prime} \times 20^{\prime}$ in size. The mean full-width at halfmaximum of the point spread function (FWHM PSF) in the final combined images is $0.84^{\prime \prime}$ in the $g$-band and $0.70^{\prime \prime}$ in the $i$-band.

Sky conditions on the night that Lac I was observed were clear. Sloan Digital Sky Survey (SDSS; Ahn et al. 2012) stars present in various images of other fields taken throughout the night were used to calculate photometric calibration coefficients that could be applied to the Lac I frames. (As mentioned in the Introduction, the Lac I field is not included in the current SDSS footprint.) The RMS scatter of the zero points calculated from individual SDSS stars within a given image taken that night ranged from 0.016-0.021 magnitude. The mean zero points for images taken of different fields and at different times of the night agreed with each other within

\footnotetext{
2 The ODI Portal, Pipeline, and Archive (ODI-PPA) system is a joint development project of the WIYN Consortium, Inc., in partnership with Indiana University's Pervasive Technology Institute (PTI) and with the National Optical Astronomy Observatory Science Data Management (NOAO SDM) Program.
}

$<0.01$ magnitude, confirming that the sky conditions were stable and the night was photometric. The photometric calibration coefficients calculated in this way were applied to all instrumental magnitudes measured via PSF photometry of the Lac I images (see next section). In addition, individual reddening corrections were calculated for each star in the Lac I images by applying the Schlafly \& Finkbeiner (2011) coefficients to the Schlegel et al. (1998) values for Galactic extinction at the position of the star. The median color excess value across the Lac I field-of-view is $E(B-V) \sim 0.14$. For the remainder of the paper, we will use final calibrated and dereddened $g_{0}$ and $i_{0}$ values.

\section{SOURCE DETECTION AND PSF PHOTOMETRY}

Point-spread-function-(PSF)-fitting photometry was performed on the final combined $g-$ and $i$-band images, using the suite of dedicated programs DAOPHOT and ALLFRAME (Stetson 1987, 1994). We began by selecting $\sim 300$ bright, non-saturated stars in each image and using them to construct a model PSF. This model PSF was then used to measure magnitudes of all sources detected in each image with peak counts at least $3 \sigma$ above the background noise level. The final photometry catalog was cleaned by retaining sources with $\chi^{2}<1.5$ (i.e., the goodness-of-fit parameter for the PSF fitting of each source) for $i_{o}>21.5$, and with a more generous cut below this value; the sharpness was additionally constrained to be $|\operatorname{sharp}|<2$ (to remove, e.g., cosmic rays or resolved background galaxies). These cuts yield a total of 33577 bona-fide stars (see Figure 1). The full photometric catalog for the Lac I $g$ and $i$ images is presented in Table 1. The catalog includes a sequence number for each star, the star's Right Ascension and Declination, the calibrated, dereddened $g_{0}$ and $i_{0}$ magnitudes and associated instrumental errors, and the calculated Galactic extinction values $\left(A_{g}\right.$ and $\left.A_{i}\right)$ that were applied.

In order to assess the true photometric uncertainties and incompleteness in the images, we injected $\sim 650,000$ artificial stars (divided into 26 separate experiments to avoid artificial crowding) distributed evenly across the frames and with colors and magnitudes in the range covered by real stars. We then performed the same detection, photometry, calibration, and dereddening steps described above to measure the magnitudes of the fake stars. The overall color-averaged $50 \%$ completeness limit is reached at $g_{0}=25.6$ and $i_{0}=24.2$, respectively. These values change to $g_{0}=25.3$ and $i_{i}=24.0$ in the innermost $\sim 3$ arcmin of Lac I due to higher stellar density there. The artificial star tests indicate that 
the photometric uncertainties are $\sim 0.1 \mathrm{mag}$ at $i_{0} \sim 23$ and $g_{0} \sim 23.5$.

\section{PROPERTIES OF LAC I}

\subsection{Color-Magnitude Diagram and Stellar Spatial Distribution}

The first two panels of Figure 2 show the dereddened CMD for stars within the half-light radius of Lac I $\left(r_{h}\right.$ $=3.24 \pm 0.21$ arcmin; see Section 4.3). The rightmost panel of Figure 2 shows a rescaled CMD of the field, for comparison. The field regions are two rectangular areas chosen near the corners of the pODI pointing, along the dwarf galaxy's minor axis and beyond $3 r_{h}$; these two regions are marked with solid lines in the left-most panel of Figure 3. The total area of the field regions is larger than the area used to produce the Lac I CMD, so we constructed the field CMD by randomly extracting a proportional number of stars from the field regions so that we were sampling equal areas of sky. The photometric uncertainties derived from the artificial star tests as described in the previous section are shown in all three panels of the figure.

Examination of Figure 2 shows that our follow-up photometry from pODI reaches $\sim 3$ magnitudes fainter than the photometry presented in the Pan-STARRS1 discovery paper (Martin et al. 2013a). A prominent red giant branch (RGB) is the main feature observed in the Lac I CMD. The RGB coincides in location with 12 Gyr old isochrones representing a range of metallicities; see Section 4.4 for more discussion. There is no overdensity of bright, blue $\left((g-i)_{0} \lesssim 0.5\right)$ sources in the Lac I CMD, and neither are there luminous asymptotic giant branch (AGB) stars, which would appear just above the TRGB. The absence of these features suggests a predominantly old nature for Lac I's stellar population. Finally, the detection of a horizontal branch/red clump, expected at $i_{0} \gtrsim 24.5$, is hampered by the rapidly decreasing completeness at these magnitudes (the $50 \%$ completeness level is marked with a dashed line in the leftmost panel of Fig. 2); we are unable to draw any firm conclusions about the presence or absence of this feature.

The top two panels of Figure 3 show the spatial distribution of the RGB stars across the pODI field-of-view. The stars used to create the upper two plots in this figure are those that appear within the RGB selection box in the leftmost panel of Figure 2. The dashed line in the left-hand panel of Figure 3 is an ellipse centered on the galaxy that marks the half-light radius derived in Section 4.3, and the arrow indicates the direction toward M31. The right-hand panel of Figure 3 shows a smoothed density map of the RGB stars, with the surface density of stars indicated by the grayscale value. It
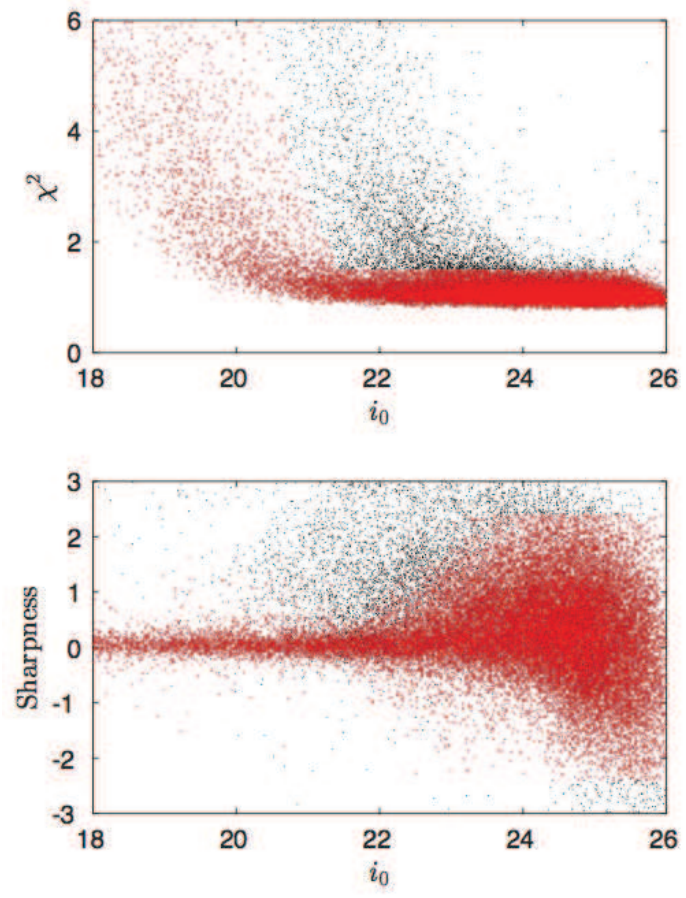

Figure 1. Source selection criteria based on the $\chi^{2}$ and sharpness parameters from the DAOPHOT photometry, as a function of dereddened $i$-band magnitude. Red sources are the ones that we retain as genuine stars, black ones are rejected.

is clear from these two upper panels of Figure 3 that there is an obvious overdensity of sources that make up the main body of Lac I, and that the galaxy has a fairly elliptical shape.

The distribution of contaminating objects in the CMD is deduced from the two rectangular field regions. As mentioned, the field regions are located beyond $3 r_{h}$ (as derived in Sec. 4.3), and while we cannot rule out the presence of Lac I stars at these radii, their number should be negligible. Examination of the field CMD in the rightmost panel of Fig. 2 illustrates that at magnitudes fainter than $i_{0} \sim 22.5$, unresolved background galaxies will be the main source of contaminating objects. At brighter magnitudes, Galactic foreground star sequences are readily apparent. For all subsequent analysis in this paper, we take into account the background/foreground contamination based on the combined CMD of the two field regions. The location of the RGB selection box marked in the CMD was chosen so that it begins at the location of the TRGB, encompasses the full width of the RGB, and cuts off at a faint mag- 


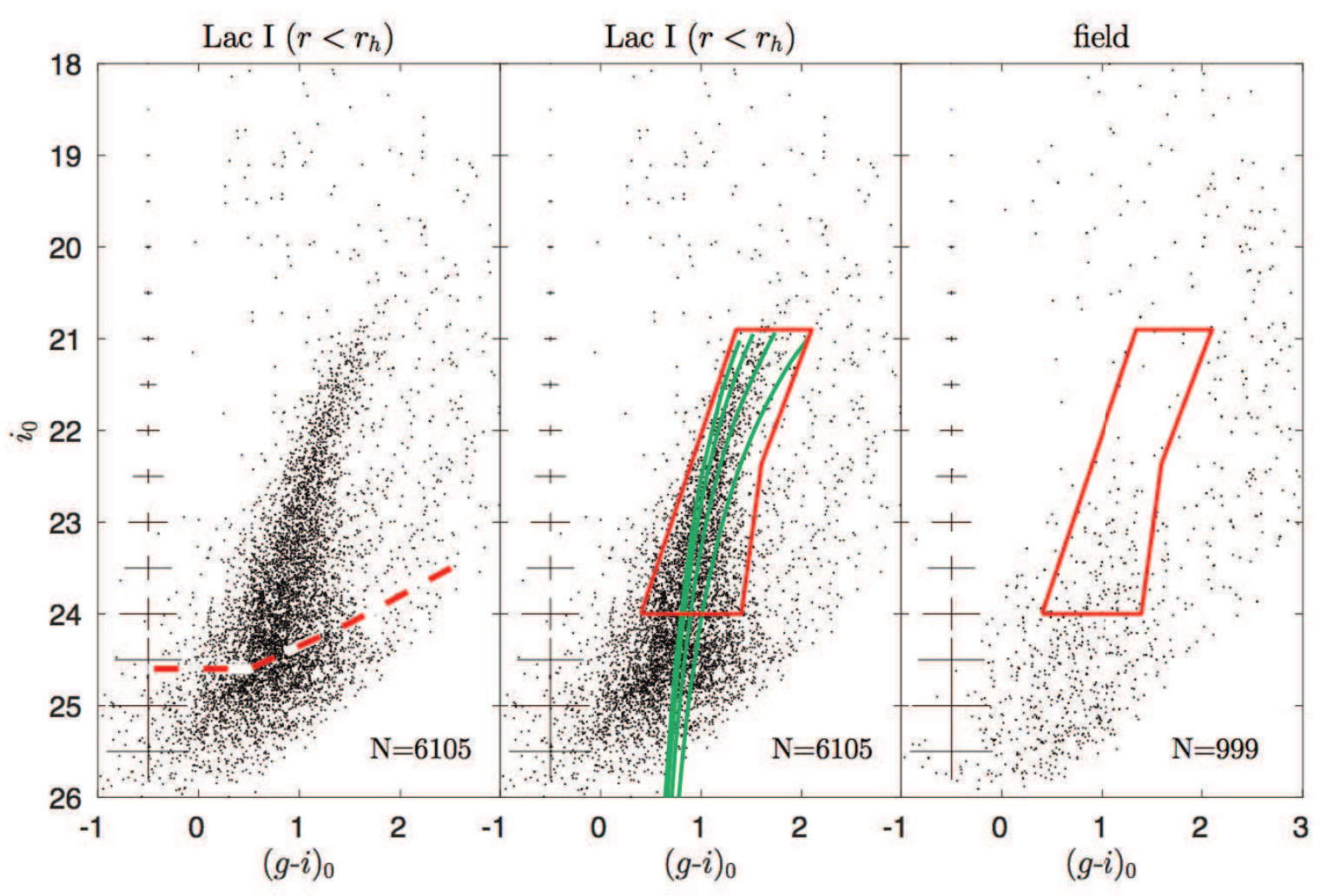

Figure 2. Dereddened CMDs of all stars within Lac I's half-light radius (left and middle panels). The red dashed line in the left panel indicates the $50 \%$ completeness level, and the photometric uncertainties derived from artificial star tests are shown in all the panels. The numbers of sources are also reported in each panel. In the middle panel, 12 Gyr Dartmouth isochrones for $[\mathrm{Fe} / \mathrm{H}]=-2.5,-2.0,-1.5$, and -1.0 (green lines) from Dotter et al. (2008) are overlaid on the data points, and the RGB selection box is marked (red polygon). The right panel shows the combined CMD of the two field regions marked in Fig. 3, rescaled to the area in the left and middle panels.

nitude limit of $i_{0}=24$ to avoid the region of the CMD with both rapidly increasing incompleteness and photometric uncertainties, as well as an increasing number of background galaxy contaminants. The magnitudes and colors of the objects that appear within these two "field" regions of the pODI images are plotted in the rightmost panel of Fig. 2.

\subsection{TRGB Distance}

The distance to Lac I is derived by applying the TRGB method (Lee et al. 1993; Rizzi et al. 2007) to our data. To accomplish this we adopt the method introduced by Makarov et al. (2006), modified following the approach of Wu et al. (2014). First, a model luminosity function is convolved with completeness, uncertainty and bias as derived from our artificial star tests; then, we fitted this model function to Lac I's luminosity function (see Figure 4), which is derived for RGB stars within $2 r_{h}$ and with a color cut of $1.2<(g-i)_{0}<1.8$ in order to consider metal-poor stars and minimize contamination from foreground Galactic stars. This procedure determines the observed magnitude at which the luminosity function has a sharp transition, which is expected for old and metal-poor RGB populations at the end of this evolutionary phase (e.g. Salaris et al. 2002). The absolute magnitude for this transition is constant, and it is computed to be $M_{i}=-3.44 \pm 0.10 \mathrm{mag}$ for the SDSS $i$-band (Bellazzini 2008). The uncertainty is computed with a Monte Carlo (MC) approach, by varying the magnitude of the observed stars within the photometric uncertainties and re-fitting the model luminosity function. From this analysis we derive a best-fit TRGB luminosity of $i_{o}=21.00 \pm 0.05 \mathrm{mag}$, which translates to a distance modulus of $(m-M)_{0}=24.44 \pm 0.11 \mathrm{mag}$, fully consistent with the $(m-M)_{0}=24.40 \pm 0.12$ mag estimate given in the Martin et al. (2013a) discovery paper. The physical distance to Lac I is thus $773 \pm 40 \mathrm{kpc}$, which 

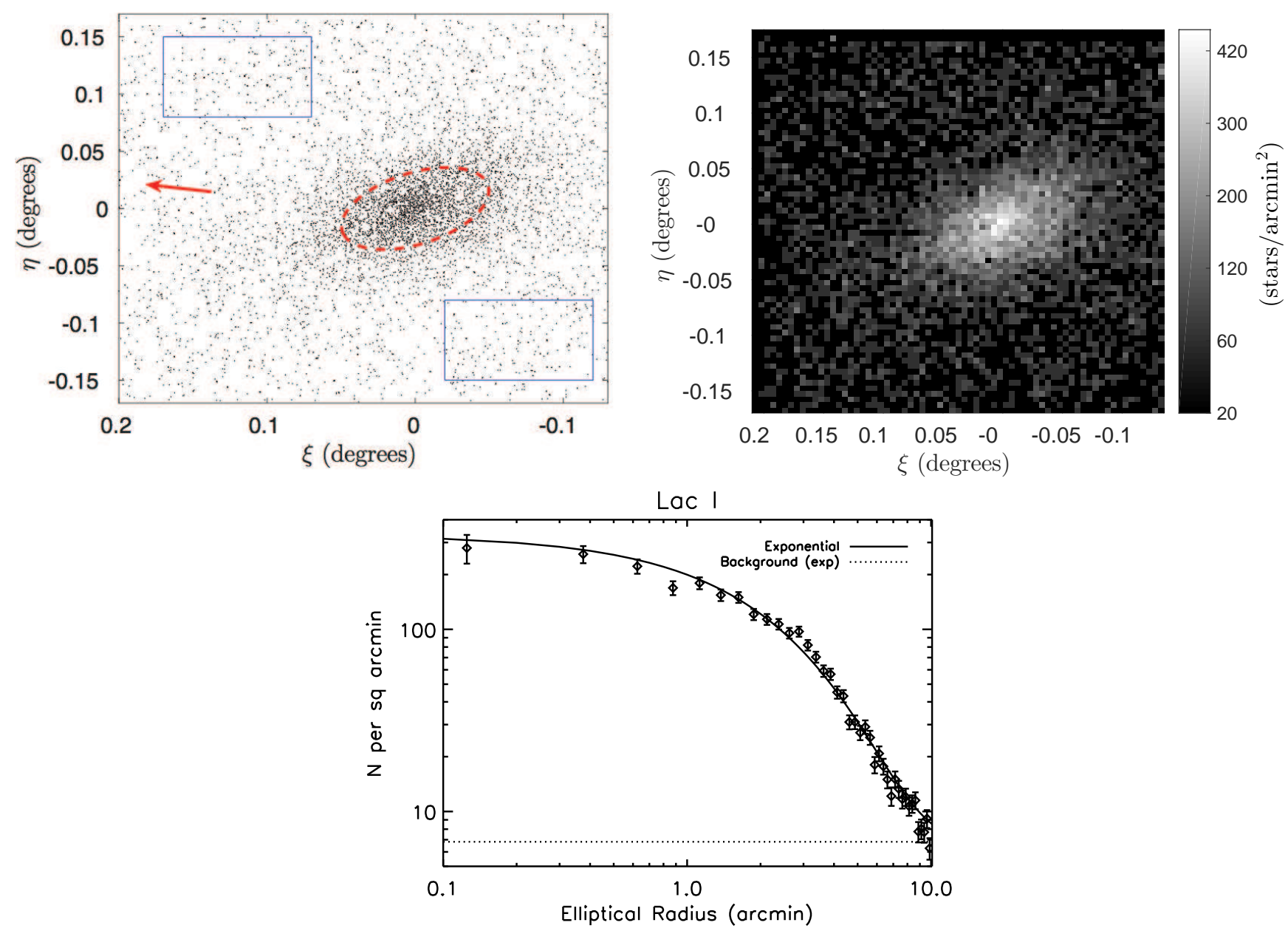

Figure 3. Top left panel. Spatial distribution of RGB stars across the pODI field-of-view (see selection box in the CMD presented in Fig. 2), in standard coordinates centered on Lac I. The dashed ellipse is drawn at the half-light radius, the rectangles are the chosen field regions and the arrow indicates the direction towards M31. Top right panel. Density map of RGB stars in Lac I (the grayscale key is shown on the right). Bottom panel. Surface density profile of RGB stars in Lac I as a function of elliptical radius. The exponential profile and background level, as derived from our maximum likelihood computation, are indicated as described in the legend. Error bars are Poisson errors on the number of objects in each radial bin.

is consistent with M31's distance. Combining this with the distance to M31 and the angular distance on the sky between the two galaxies yields a 3D distance for Lac I of $264 \pm 6 \mathrm{kpc}$ from M31.

\subsection{Structural Parameters, Luminosity, and HI Gas Content}

We fitted an exponential profile distribution to the surface density profile of Lac I, utilizing the maximum likelihood technique described by Martin et al. (2008), as implemented by Sand et al. (2012). While real satellites often have a complexity that cannot be encapsulated by parameterized models such as the exponential profile, such profiles are useful for quantifying the galaxies' basic structural properties and for direct comparison with other results. The stars selected for the structural analysis are drawn from the RGB selection box seen in
Figure 2, which includes a limiting magnitude of $i_{0}=24$ mag so as to avoid any adverse effects from crowding at faint magnitudes. The free parameters for our exponential profile model are: the central position $\left(\alpha_{0}, \delta_{0}\right)$, ellipticity $(\epsilon$, which is defined as $1-b / a$, where $b$ is the scale length along the minor axis and $a$ is the scale length along the major axis), position angle (PA; $\theta)$, half-light radius $\left(r_{h}\right)$ and background surface density $\left(\Sigma_{b}\right)$. Uncertainties on each parameter were calculated via 1000 bootstrap resamples of the data, from which $68 \%$ confidence limits were derived. The resulting structural parameters for Lac I are presented in Table 2, along with the other properties we measure for the galaxy. The probability distribution functions derived from the maximum likelihood analysis are shown in Figure 5. 


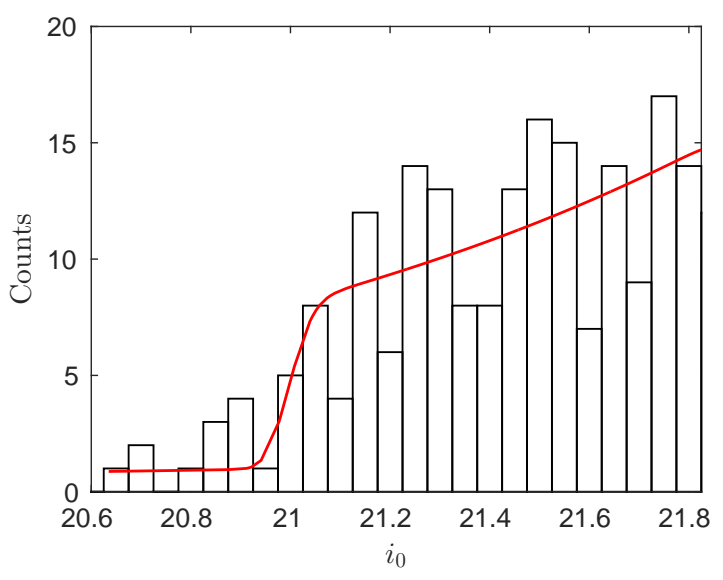

Figure 4. Luminosity function of Lac I's RGB stars within $2 r_{h}$ and with a color cut of $1.2<(g-i)_{0}<1.8$ : the red line is the best-fitting model luminosity function applied in order to derive the TRGB luminosity.

Our structural parameters are in agreement with the discovery data of Martin et al. (2013a) to within the uncertainties, although our derived half-light radius is smaller and slightly more than 1- $\sigma$ discrepant. The structural parameters presented here should not be biased given the depth of the data, the stellar density contrast compared to the background, and the field of view of the pODI data; our data set meets all of the criteria determined by Muñoz et al. (2012) for deriving accurate structural parameters using our maximum likelihood technique.

We show a one-dimensional representation of our bestfitting stellar profile in the bottom panel of Figure 3. While the binned data points are not used to fit stellar profiles - as the maximum likelihood technique uses the two dimensional unbinned distribution of stars - the agreement between the best-fitting model stellar profile and the data points is excellent, with only minor deviations from an exponential profile.

To measure the total magnitude of Lac I, we first sum the flux within one half-light radius using the RGB stars within the CMD selection box shown in Figure 2. From this, we subtract off the flux from foreground and background sources using the field regions marked in the upper left panel of Figure 3 and rescaling to match the area used for Lac I. We then multiply the remaining flux by a factor of two to account for the flux outside of Lac I's half light radius. We correct for stars below our detection limit by using several metal poor $([\mathrm{Fe} / \mathrm{H}]=$ -1.5 to -2.0$)$ and old (10-13 Gyr) PARsec luminosity functions (Bressan et al. 2012). From this, we measure $M_{g}=-11.3 \pm 0.2 \mathrm{mag}$ and $M_{i}=-12.0 \pm 0.2 \mathrm{mag}$, and convert this to $M_{V}=-11.4 \pm 0.3 \mathrm{mag}$ using the filter transformation of Veljanoski et al. (2013). Our total magnitude is consistent within the uncertainties with the value presented in the Martin et al. (2013a) discovery paper $\left(M_{V}=-11.7 \pm 0.7 \mathrm{mag}\right)$, although our uncertainty is a factor of two smaller. The central surface brightness of Lac I is calculated using the total magnitude and the best-fitting exponential profile described earlier, and is $\mu_{V, 0}=24.8 \pm 0.3 \mathrm{mag} / \mathrm{arcsec}^{2}$. Our measured $\mu_{V, 0}$ value is a full magnitude brighter than the corresponding value from Martin et al. (2013a) $\left(\mu_{V, 0}\right.$ $\left.=25.8 \pm 0.8 \mathrm{mag} / \operatorname{arcsec}^{2}\right)$, although the numbers are in agreement to within the uncertainties. As is the case with the total magnitude, the uncertainty on our central surface brightness measurement is substantially reduced compared to the previous measurement. (Note that this increase in surface brightness is not unexpected, since our derived half-light radius is smaller.) The derived magnitudes and surface brightness values are included in Table 2.

We investigated the neutral gas content of Lac I by searching for HI emission along the line-of-sight to the galaxy in publicly-available Effelsberg Bonn HI Survey data (EBHIS; Winkel et al. 2016), smoothed to a spectral resolution of $15 \mathrm{~km} \mathrm{~s}^{-1}$. We find no $\mathrm{HI}$ emission in the smoothed spectrum at the systemic velocity for Lac I reported by Martin et al. (2014), and compute a $5-\sigma$, single-channel upper limit on the HI mass within $6^{\prime}\left(1.85 r_{h}\right)$ of $M_{H I}^{l i m}=1.9 \times 10^{5} M_{\odot}$. This implies $M_{H I} / L_{V}<0.06 M_{\odot} / L_{\odot}$ for Lac I (see 2), and therefore that this galaxy is gas-poor like the other dwarf spheroidal galaxies around the Milky Way and M31 (Grcevich \& Putman 2009; Spekkens et al. 2014).

\subsection{Photometric Metallicity Distribution Functions}

We derive photometric metallicities for each individual RGB star that is located within our RGB selection box (marked in Fig. 2) and also has $i_{0}<23$ mag. Applying this additional faint-magnitude cut reduces contamination significantly and keeps the photometric uncertainties below $0.1 \mathrm{mag}$ in magnitude and $0.2 \mathrm{mag}$ in color, to ensure that we are using the bestquality photometric data to yield metallicity information. Photometric metallicities are obtained by linearly interpolating among stellar isochrones with a fixed aged of $12 \mathrm{Gyr}$ and varying metallicity $([\mathrm{Fe} / \mathrm{H}]=-2.5$ to -1.0; Dotter et al. 2008). Under the assumption that the RGB width is mainly driven by metallicity rather than age, the color of a RGB star will correspond to an isochrone with a given metallicity (see Fig. 2, and Crnojević et al. 2010 for details). This approximation is valid in the absence of significant intermediate-age populations, which appears to be a safe assumption for 

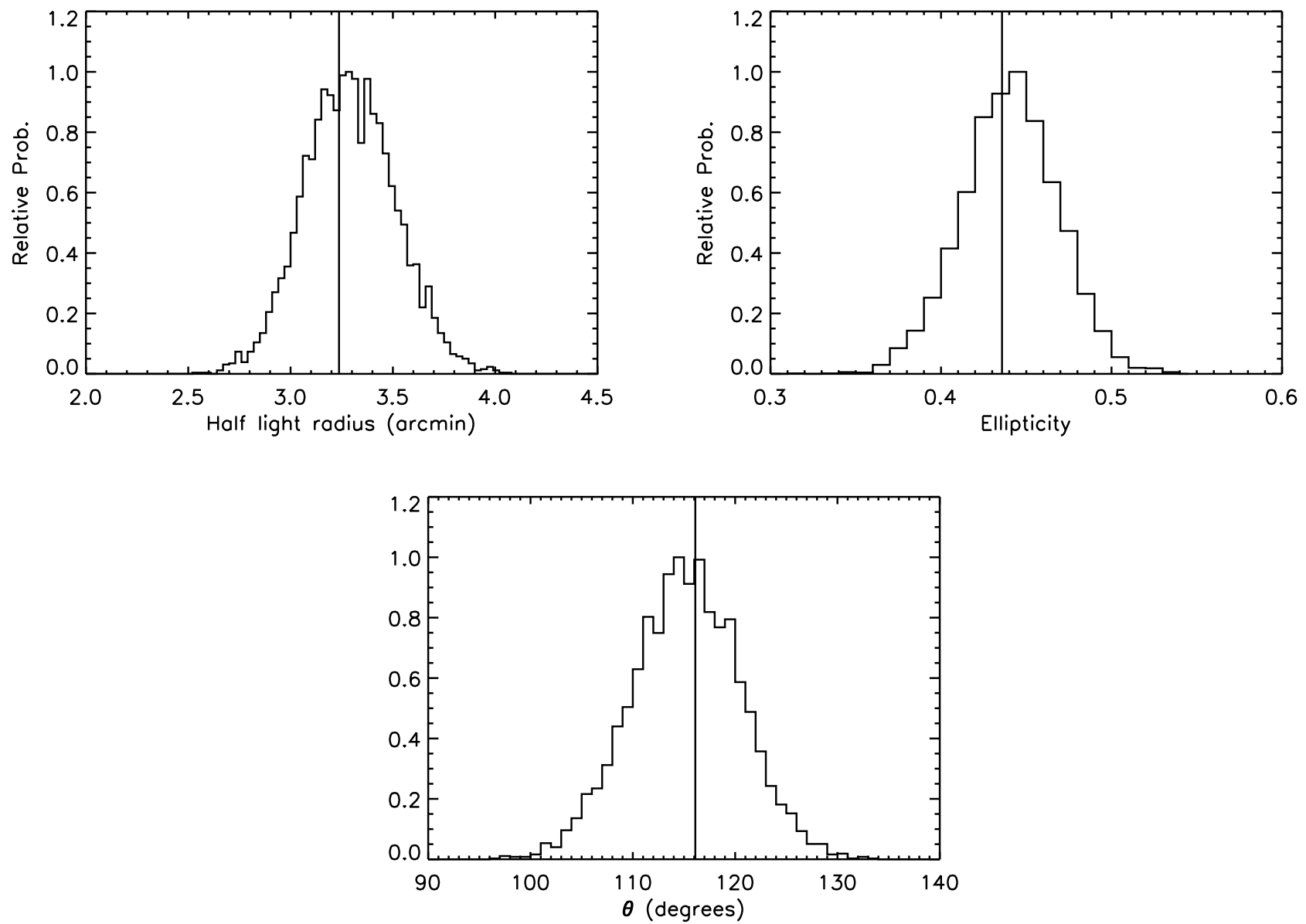

Figure 5. Probability distribution functions for the structural parameters derived from the maximum likelihood analysis described in Section 4.3.

Lac I, based on the appearance of the CMD. (We do note, however, that Martin et al. (2014) state that they identified "a handful" of carbon stars in Lac I based on the shape of a few of the stellar spectra they obtained, and that the presence of these stars may suggest that Lac I has at least a modest intermediate-age population.) This type of approach provides robust results in terms of relative quantities such as the derivation of radial metallicity trends (VandenBerg et al. 2006; Crnojević et al. 2014). We also explore the effect that a slightly younger average age would have on the derived metallicities: if we adopt isochrones with a fixed $8 \mathrm{Gyr}$ age instead of $12 \mathrm{Gyr}$, the metallicity values obtained for Lac I (see below) become more metal-rich by about 0.2 dex. (See also Crnojević et al. 2010 for an extensive discussion on the possible effects of prolonged star formation on the derivation of photometric metallicities.)

In Figure 6 we show the resulting metallicity distribution functions (MDFs) for stars separated into three regions based on their elliptical radius. To calculate the elliptical radius $(r)$ for each star, we first transform the star's equatorial coordinates into standard coordinates ( $\xi$ and $\eta$; see Figure 3 ) on a plane tangential to the center of Lac I. We then take these coordinates and use them as the $X_{i}$ and $Y_{i}$ coordinates in the definition of elliptical radius given in Martin et al. (2008) (their Equation 4). The three panels in Figure 6 show the $[\mathrm{Fe} / \mathrm{H}]$ distribution of stars with $r<r_{h}$ (top panel), $r_{h}<r<2 r_{h}$ (middle panel), and $r>2 r_{h}$ (bottom panel). The outermost bin includes stars out to a radius of 9 arc minutes, i.e., the largest ellipse fully enclosed within the pODI images (which is just short of $3 r_{h}$ ). We do not correct the derived MDFs for incompleteness, since the RGB selection box is $\sim 97 \%$ complete down to $i_{0}<23$. To correct for contamination in the stellar sample, we derive an "MDF" for the field regions as well (strictly speaking, contaminating sources are not RGB stars belonging to the same population, so the derived metallicities are not meaningful), and subtract it from the Lac I MDFs after rescaling it to the area of each radial bin. The percentage of contaminants in the three radial bins is $\sim 2 \%$, $9 \%$, and $28 \%$, respectively. 
We used the information about contamination gleaned from the field "MDF" to correct not only the Lac I MDF data in histogram form but also the individual photometric metallicity values of the stars in the three radial regions shown in Figure 6. We corrected the individual photometric metallicity measurements by randomly removing the appropriate number of stars falling within a given metallicity range in each radial region. In those cases when the number of contaminants in a given metallicity and radial range was a fraction, the value was rounded to the nearest integer, and then that number of stars was randomly removed from the appropriate data file.

We used the contamination-corrected MDF data to calculate the median metallicity of the stars in the three radial ranges shown in Figure 6. Uncertainties on these median $[\mathrm{Fe} / \mathrm{H}]$ values were derived with a set of $\mathrm{MC}$ experiments. First, the magnitude and color of each star in the MDF was varied within the photometric uncertainties for that star. The metallicity was rederived for that magnitude and color using the same method as for the original data (interpolation from the isochrones). We did this 1000 times for each star and calculated the median $[\mathrm{Fe} / \mathrm{H}]$ value for each realization, and within each radial region. The spread of $[\mathrm{Fe} / \mathrm{H}]$ values computed from the MC simulations yields an estimate of the uncertainty in the $[\mathrm{Fe} / \mathrm{H}]$ derived from the original data.

The median metallicities for the contaminationcorrected MDFs in the three radial regions are $[\mathrm{Fe} / \mathrm{H}]=$ $-1.66 \pm 0.03$ dex for the innermost radial region (inside $\left.r_{h}\right),[\mathrm{Fe} / \mathrm{H}]=-1.69 \pm 0.03$ for stars with $r_{h}<r<2 r_{h}$, and $[\mathrm{Fe} / \mathrm{H}]=-1.72 \pm 0.05$ for stars in the outer radial region (outside $2 r_{h}$ ). These values are marked in the histogram plots in Figure 6. Combining all of the $[\mathrm{Fe} / \mathrm{H}]$ values for stars within $2 r_{h}$ yields a median $[\mathrm{Fe} / \mathrm{H}]$ of -1.68 , and combining all three radial regions yields the same median value; we have listed this value in Table 2 .

The median $[\mathrm{Fe} / \mathrm{H}]$ values in all three of the radial regions are more metal-rich than the $[\mathrm{Fe} / \mathrm{H}]$ value of $-2.0 \pm 0.1$ derived by Martin et al. (2014) from their spectroscopic observations of 126 RGB stars in Lac I, although in some cases the difference is not significant when the uncertainties on these values are taken into account. The fixed-age approximation inherent to the photometric metallicity derivation method can only partly explain this discrepancy. For the discrepancy with the Martin et al. (2014) data set to be alleviated, their spectroscopic sample would need to have missed the majority of the metal-rich subsample highlighted by our MDFs. A close inspection of our MDFs suggests that there could be two metallicity peaks, at $[\mathrm{Fe} / \mathrm{H}] \sim-1.8$ and $[\mathrm{Fe} / \mathrm{H}] \sim-1.4$ respectively, which are most pro- nounced in the innermost radial bin (top panel of Fig. 6). The distinction fades off in the middle bin, while the last adopted bin contains few stars due to the rapidly decreasing stellar density beyond $r=2 r_{h}$, thus any possible trend gets lost in the noise. We stress that the radial MDFs have a comparable shape when derived under the assumption of a fixed 8 Gyr age.

We carried out the following steps to investigate whether the apparent multiple peaks in the MDFs might actually be statistically significant. The contaminationcorrected MDF data (i.e., the individual photometric metallicity values of the stars in the three radial regions shown in Figure 6, corrected for contamination) were evaluated with a series of statistical tests to assess the likelihood that a mixture of one, two, or three Gaussian distributions can produce the observed values. We used a parametric bootstrap Anderson-Darling (A-D) test (Anderson \& Darling 1952) to independently test each Gaussian mixture as a potential fit to the MDFs. First, a set of one to three Gaussian functions was fitted to the input data, and best-fitting means, dispersions, and mixing proportions for each Gaussian were returned. The original data were evaluated against the candidate mixture model using the A-D test. Next, 1000 synthetic data sets with the same parameters as the best-fitting mixture model were produced and evaluated with the A-D test. The A-D score derived from the observed data was then compared to the distribution of bootstrapped A-D scores. This testing procedure indicated with high confidence that the observed MDFs for all three radial regions are not likely to be drawn from a unimodal or bimodal Gaussian distribution. The data in the outermost radial region $\left(r>2 r_{h}\right)$ is also not likely to be drawn from a trimodal Gaussian distribution. The tests also indicated, however, that there is a high probability that the MDF data for the inner two radial regions $\left(r<r_{h}\right.$ and $\left.r_{h}<r<2 r_{h}\right)$ is drawn from a mixture of three Gaussian distributions. The peak values (and dispersions) of the best-fitting Gaussian functions are $[\mathrm{Fe} / \mathrm{H}]=-1.3(0.1),-1.8(0.3)$, and $-2.4(0.1)$ and mixing fractions of $31 \%, 63 \%$, and $6 \%$, respectively for the stars inside the half-light radius $r_{h}$. The corresponding values for stars with radii between $r_{h}$ and $2 r_{h}$ are $[\mathrm{Fe} / \mathrm{H}]=-1.4(0.1),-1.8(0.2)$, and $-2.4(0.1)$ and mixing fractions of $25 \%, 71 \%$, and $5 \%$, respectively.

We performed additional analysis steps to determine whether the parametric bootstrap A-D test was identifying multi-modality simply because of the noise in the data or biases introduced during the conversion from photometric measurements to metallicities. We generated a synthetic MDF by populating an isochrone to 
produce a 12 -Gyr population with $[\mathrm{Fe} / \mathrm{H}] \sim-1.7$. We convolved this catalog with the photometric errors from our data set to obtain an "observed" CMD. We derived metallicities for RGB stars within the upper $\sim 2$ magnitudes of the synthetic RGB, just as was done for the real data. We then carried out the bootstrap testing procedure on the synthetic $[\mathrm{Fe} / \mathrm{H}]$ values and found that the best-fitting unimodal, bimodal, and trimodal Gaussian distributions were all unlikely to reproduce the particular distribution of the test data set at a high confidence level $(99.999 \%, 98.3 \%$, and $99.5 \%$ for the unimodal, bimodal, and trimodal distributions respectively). Since the bootstrap testing rejected not only the bimodal and trimodal mixture models but also the single-metallicity model, we can only infer from this that the observed trimodality that is implied by the A-D testing on the real data may not be genuine either. Our overall conclusion based on this analysis is that although it is possible that a dwarf galaxy with the properties of Lac I could indeed have multiple stellar populations, our photometric metallicities are unable to unambiguously demonstrate their presence or absence.

The spectroscopic metallicity value reported by Martin et al. (2014) separates Lac I from the bulk of Local Group dwarfs on a luminosity-metallicity plot (Fig. 4 in their paper, which shows the positions of M31 satellite dwarf galaxies on the luminosity-metallicity (LZ) relation derived by Kirby et al. (2013) from nearby dwarfs), i.e., it is too metal-poor for its luminosity. We can take our updated absolute magnitude measurement, $M_{V}=-11.4 \pm 0.3$, and our median photometric metallicity estimate of $[\mathrm{Fe} / \mathrm{H}] \sim-1.68$ (from all of the stars in the contamination-corrected MDFs), and compare these values to the Kirby et al. (2013) L-Z relation (their Equation 3). Assuming this relation, the predicted $[\mathrm{Fe} / \mathrm{H}]$ value for Lac I given its luminosity is -1.54. The RMS scatter of the Kirby et al. relation is $0.16 \mathrm{dex}$, so our photometric metallicity value is, interestingly, more in line with the expected value.

Lastly, we also looked for the presence of a metallicity gradient in Lac I. We used the photometrically-derived metallicities for stars within one and two $r_{h}$. We did not correct for contamination for this experiment because the field-star contamination would be only $2 \%$ within $r_{h}$ and $8 \%$ within $2 r_{h}$. The measured metallicity gradient in our data is $d[\mathrm{Fe} / \mathrm{H}] / d\left(r / r_{h}\right)=0.0028 \pm 0.0029$ and $-0.0014 \pm 0.0065$ dex per $r_{h}$, respectively, which is consistent with a flat metallicity profile. The lack of a gradient has also been observed in some dwarf galaxies of similar luminosity beyond the Local Group (e.g., Crnojević et al. 2010), which may suggest that the galaxies are not massive enough to favor a second episode of star formation and thus a distinct and more metal-rich population in their central regions.

\section{SUMMARY AND MAIN CONCLUSIONS}

In this paper we presented results from deep, widefield WIYN pODI $g, i$ imaging of the M31 dwarf satellite galaxy Lac I (And XXXI), acquired in order to investigate the galaxy's structure, stellar populations, and metallicity. The CMD of this galaxy is dominated by an old ( 12 Gyr) stellar population and no intermediateage stars (AGB stars) are apparent in our data. We trace the RGB stars in the Lac I images to a radius of $\sim 10 \operatorname{arcmin}(\sim 2.25 \mathrm{kpc})$ from the galaxy center and use them to derive a distance to the galaxy as well as its structural properties. Our measured TRGB distance of $773 \pm 40 \mathrm{kpc}$ agrees with the Pan-STARRS discovery paper distance (Martin et al. 2013a). Our derived 3D distance for Lac I to M31 is $264 \pm 6 \mathrm{kpc}$ and we confirm the finding by Martin et al. (2013a) that Lac I is in the far western outskirts of the Andromeda galaxy halo.

Despite its relatively late discovery and distant location from its host galaxy, Lac I seems in other ways to be a fairly typical Local Group dwarf spheroidal galaxy. Our derived half-light radius $r_{h}$ and associated uncertainty for the galaxy, $728 \pm 47 \mathrm{pc}$, are smaller than the Pan-STARRS1-measured value $\left(912_{-93}^{+124} \mathrm{pc}\right)$, although they agree within the uncertainties. Brasseur et al. (2011) used the measured $V$-band absolute magnitudes and half-light radii of dSph satellites of the Galaxy and M31 and found that they follow a well-defined sizeluminosity relation and that the relations for each galaxy (the Milky Way and M31) are statistically the same. Given its measured $M_{V}$ of -11.4 from our pODI data, the derived half-light radius of Lac I is right in line with the value predicted by the Brasseur et al. (2011) relation for Andromeda dSph galaxies, $685_{-220}^{+213} \mathrm{pc}$. Our measured ellipticity value, $\epsilon=0.44 \pm 0.03$ is almost identical to the Pan-STARRS1 value, but with a formal uncertainty that is a factor of two smaller. This ellipticity is within the usual range for dSph satellites of Andromeda; for the dSph galaxies in the M31 sub-group, the range of ellipticities is $0.13-0.56$, with a mean $\epsilon$ of 0.31 and a dispersion of 0.12 (McConnachie 2012). We have confirmed that this galaxy is gas-poor, with an upper limit on the HI gas to $L_{V}$ ratio that is comparable to the most sensitive limits for dSph galaxies orbiting the Milky Way (Spekkens et al. 2014).

Our investigation of the MDF of Lac I - which we derived from photometry of the RGB stars with $i_{0}<23$ mag - indicates that this galaxy is metal-poor, with a median $[\mathrm{Fe} / \mathrm{H}]$ of $-1.68 \pm 0.03$ for stars within $2 r_{h}$, but a broad range of stellar metallicities, from $[\mathrm{Fe} / \mathrm{H}] \sim-1$ to 
-2.5. Although the appearance of the MDFs in certain radial regions seems to suggest the presence of multiple metallicity peaks, statistical testing performed on the distributions indicates that the multi-modality may not be real. We find no evidence for a radial gradient in our photometric metallicities. Lastly, we note that the median metallicity derived from our RGB star photometry is higher than the median metallicity of $[\mathrm{Fe} / \mathrm{H}]=$ $-2.0 \pm 0.1$ measured by Martin et al. (2014) from their spectroscopic observations of 126 bright $\left(i_{P 1,0} \lesssim 20.5\right)$ RGB stars in Lac I, and the somewhat higher metallicity we measure moves Lac I closer to the expected $L-Z$ relation for dwarf galaxies (Kirby et al. 2013). The causes of the differences in the photometric versus spectroscopic metallicities are not obvious and seem to warrant further investigation.

We are grateful to the staff of the WIYN Observatory and Kitt Peak National Observatory for their assistance with using WIYN and the pODI camera to obtain the data used for this study. We thank the staff members at WIYN, NOAO, and Indiana University Pervasive Technology Institute who designed, im- plemented, and helped us work with the ODI-PPA and produce high-quality science results with our pODI images. We also thank the anonymous referee for helpful comments that improved the paper. K.L.R. was supported by NSF Faculty Early Career Development (CAREER) award AST-0847109 or by NSF Astronomy \& Astrophysics Research Grant number AST-1615483 during the time when this research was carried out. D.J.S. acknowledges support from NSF Astronomy \& Astrophysics Research Grant number AST-1412504. S.J. acknowledges support from the Australian Research Council's Discovery Project funding scheme (DP150101734). K.S. acknowledges support from the Natural Sciences and Engineering Research Council of Canada (NSERC). This research has made use of the NASA/IPAC Extragalactic Database (NED) which is operated by the Jet Propulsion Laboratory, California Institute of Technology, under contract with the National Aeronautics and Space Administration.

Software: ODI-PPA (Gopu et al. 2014), QuickReduce pipeline (Kotulla 2014), DAOPHOT (Stetson 1987), ALLFRAME (Stetson 1994)

\section{REFERENCES}

Adams, E.A.K., Giovanelli, R., \& Haynes, M.P. 2013, ApJ, 768,77

Ahn, C., Alexandroff, R., Allende Preito, C., et al. 2012, ApJS, 203, 21

Anderson, T. \& Darling, D. 1952, Ann. Math. Statist. 23,

No. 2, pp. 193-212

Bechtol, K., et al. 2015, ApJ, 807, 50

Bell, E.F., Slater, C.T., \& Martin, N.F. 2011, ApJ, 742, L15

Bellazzini, M. 2008, MmSAI, 79, 440

Belokurov, V. et al. 2007, ApJ, 654, 897

Brasseur, C.M., Martin, N.F., Maccio, A.V., Rix, H.-W., \&

Kang, X. 2011, ApJ, 743, 179

Bullock, J.S. \& Johnston, K.V. 2005, ApJ, 635, 931

Bressan, A., Marigo, P., Girardi, L. et al. 2012, MNRAS,

427,127

Crnojević, D., Grebel, E.K., \& Koch, A. 2010, A\&A, 516, 85

Crnojević, D., Ferguson, A.M.N., Irwin, M.J., McConnachie, A.W., Bernard, E.J., Fardal, M.A., Ibata, R.A., Lewis, G.F., Martin, N.F., Navarro, J.F., Noel, N.E.D., \& Pasetto, S. 2014, MNRAS, 445, 3862

Dotter, A., Chaboyer, B., Jevremović, D., Kostov, V.,

Baron, E., \& Ferguson, J.W. 2008, ApJS, 178, 89

Grcevick, J. \& Putman, M.E. 2009, ApJ, 696, 385
Gopu, A., Hayashi, S., Young, M.D., Harbeck, D.R., Boroson, T., Liu, W., Kotulla, R., Shaw, R., Henschel, R., Rajagopal, J., Stobie, E., Knezek, P., Martin, R.P., Archbold, K. 2014, Proceedings of the SPIE, Vol. 9152, p. $91520 \mathrm{E}$

Harbeck, D.R., Boroson, T., Lesser, M., Rajagopal, J., Yeatts, A., Corson, C., Liu, W., Dell'Antonio, I., Kotulla, R., Ouellette, D., Hooper, E., Smith, M., Bredthauer, R., Martin, P., Muller, G., Knezek, P., \& Hunten, M. 2014, Proceedings of the SPIE, Vol. 9147, p. 9147E

Ibata, R.A., Lewis, G.F., Conn, A.R., Irwin, M.J., McConnachie, A.W., Chapman, S.C., Collins, M.L., Fardal, M., Ferguson, A.M.N., Ibata, N.G., Mackey, A.D., Martin, N.F., Navarro, J., Rich, R.M., Valls-Gabaud, D., Widrow, L.M. 2013, Nature, 493, 62 Janesh, W.F., Rhode, K.L., Salzer, J.J., Janowiecki, S., Adams, E.A.K., Haynes, M.P., Giovanelli, R., Cannon, J.M., \& Munoz, R.R. 2015, ApJ, 811, 35

Jenkins, A., Frenk, C.S., White, S.D.M., Colberg, J.M., Cole, S., Evrard, A. E., Couchman, H.M.P., Yoshida, N. 2001, MNRAS, 321, 372

Kim, D., Jerjen, H., Mackey, D., Da Costa, G.S., Milone, A.P. 2015, ApJ, 804, L44

Kirby, E., Cohen, J., Guhathakurta, P., Cheng, L., Bullock, J.S., \& Gallazzi, A. 2013, ApJ, 779, 102 
Klypin, A., Kravtsov, A. V., Valenzuela, O., \& Prada, F. 1999, ApJ, 522, 82

Koposov, S.E., Belokurov, V., Torrealba, G., \& Evans, N.W. 2015, ApJ, 805, 130

Kotulla, R. 2014, in Astronomical Data Analysis Software and Systems XXIII, Astronomical Society of the Pacific Conference Series, Vol. 485, eds. N. Manset \& P. Forshay, 375

Lee, M.G., Freedman, W.L., \& Madore, B.F. 1993, ApJ, 417, L553

Makarov, D., Makarova, L., Rizzi, L., Tully, R.B., Dolphin, A.E., Sakai, S., \& Shaya, E.J. 2006, AJ, 132, 2729

Martin, N., et al. 2006, MNRAS, 371, 1983

Martin, N. F., de Jong, J. T. A., \& Rix, H.-W. 2008, ApJ, 684,1075

Martin, N.F., Slater, C.T., Schlafly, E.F., Morganson, E., Rix, H.-W., Bell, E.F., Laevens, B.P.M., Bernard, E.J., Ferguson, A.M.N., Finkbeiner, D.P., Burgett, W.S., Chambers, K.C., Hodapp, K.W., Kaiser, N., Kudritzki, R.-P., Magnier, E.A., Morgan, J.S., Price, P.A., Tonry, J.L., \& Wainscoat, R.J. 2013, ApJ, 772, 15

Martin, N.F., Schlafly, E.F., Slater, C.T., Bernard, E.J., Rix, H.-W., Bell, E.F., Ferguson, A.M.N., Finkbeiner, D.P., Laevens, B.P.M., Burgett, W.S., Chambers, K.C., Draper, P.W., Hodapp, K.W., Kaiser, N., Kudritzki, R.-P., Magnier, E.A., Metcalfe, N., Morgan, J.S., Price, P.A., Tonry, J.L., Wainscoat, R.J., \& Waters, C. 2013, ApJ, 779, L10

Martin, N.F., Chambers, K.C., Collins, M.L.M., Ibata, R.A., Rich, R.M., Bell, E.F., Bernard, E.J., Ferguson, A.M.N., Flewelling, H., Kaiser, N., Magnier, E., Tonry, J.L., \& Wainscoat, R.J. 2014, ApJ, 793, L14
McConnachie, A.W., et al. 2009, Nature, 461, 66

McConnachie, A.W., 2012, AJ, 144, 4

Moore, B., Ghigna, S., Governato, F., Lake, G., Quinn, T., Stadel, J., \& Tozzi, P. 1999, ApJ, 524, L19

Muñoz, R.R., Padmanabhan, N., \& Geha, M. 2012, ApJ, 745,127

Rizzi, L., Tully, R.B., Makarov, D., Makarova, L., Dolphin, A.E., Sakai, S., \& Shaya, E.J. 2007, ApJ, 661, 815

Salaris, M., Cassisi, S., \& Weiss, A. 2002, PASP, 114, 375

Sand, D.J., Strader, J., Willman, B., Zaritsky, D., McLeod, B., Caldwell, N., Seth, A., \& Olszewski, E. 2012, ApJ, 756,79

Schlafly, E.F. \& Finkbeiner, D.P. 2011, ApJ, 737, 103

Schlegel, D.J., Finkbeiner, D.P., \& Davis, M. 1998, ApJ, 500,525

Simon, J.D. \& Geha, M. 2007, ApJ, 670, 313

Spekkens, K., Urbancic, N., Mason, B.S., Willman, B., \& Aguirre, J.E. 2014, ApJ, 795, L5

Stetson, P.B. 1987, PASP, 99, 191

Stetson, P.B. 1994, PASP, 106, 250

VandenBerg, D.A., Bergbusch, P.A., \& Dowler, P.D. 2006, ApJS, 162, 375

Veljanoski, J., Ferguson, A. M., Huxor, A.P. et al. 2013, MNRAS, 435, 3654

Willman, B. et al. 2005, ApJ, 626, L85

Winkel, B., Kerp, J., Floer, L., Kalberla, P.M.W., Ben Bekhti, N., Keller, R., \& Lenz, D. 2016, A\&A, 585, 41

Wu, P., Tully, R.B., Rizzi, L., Dolphin, A.E., Jacobs, B.A., \& Karachentsev, I.D. 2014, AJ, 148, 7 

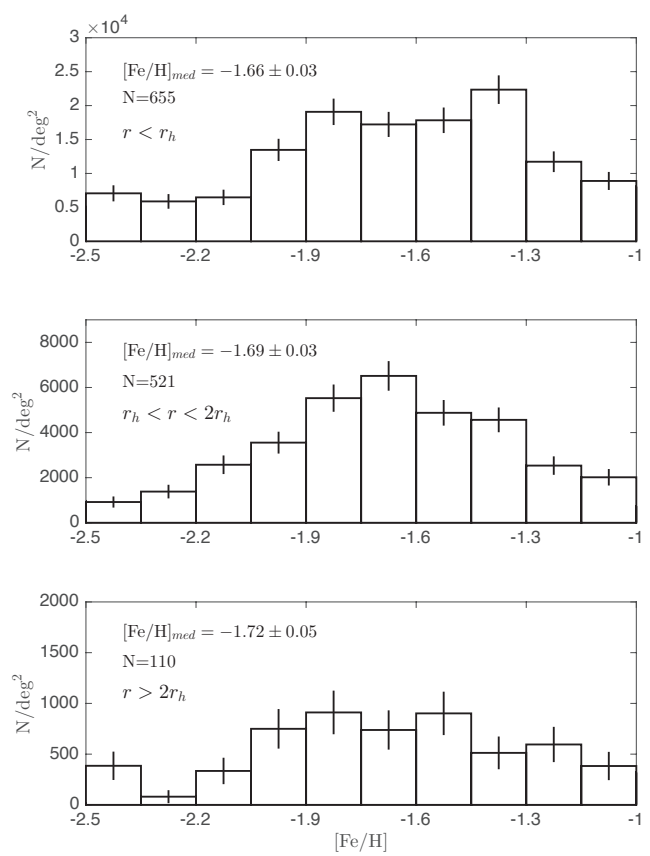

Figure 6. Photometric metallicity distribution functions (per unit area) for stars that lie within the RGB selection box and have $i_{0}$ magnitude brighter than 23.0. The three panels show MDFs for stars that meet these criteria, divided into three spatial regions: within one $r_{h}$ of the galaxy center (top), between one and two $r_{h}$ from the center, and outside of two $r_{h}$ (bottom). The number of stars per radial bin and the median metallicity are also reported. These MDFs have been corrected for contamination, as described in Section 4.4.

Table 1. Photometry of Resolved Stars in the WIYN pODI Images of Lac I

\begin{tabular}{ccccccccc}
\hline \hline$\#$ & $\alpha(2000)$ & $\delta(2000)$ & $g_{o}$ & $\sigma_{g}$ & $A_{g}$ & $i_{o}$ & $\sigma_{i}$ & $A_{i}$ \\
& $(\mathrm{deg})$ & $(\mathrm{deg})$ & $(\mathrm{mag})$ & $(\mathrm{mag})$ & $(\mathrm{mag})$ & $(\mathrm{mag})$ & $(\mathrm{mag})$ & $(\mathrm{mag})$ \\
\hline 1 & 344.37250 & +41.458038 & 16.73 & 0.01 & 0.52 & 15.64 & 0.01 & 0.27 \\
2 & 344.37418 & +41.425993 & 25.58 & 0.29 & 0.53 & 24.53 & 0.16 & 0.27 \\
3 & 344.37427 & +41.425386 & 24.87 & 0.12 & 0.53 & 25.14 & 0.20 & 0.27 \\
4 & 344.37434 & +41.436219 & 24.95 & 0.11 & 0.53 & 24.22 & 0.08 & 0.27 \\
5 & 344.37458 & +41.422852 & 23.43 & 0.04 & 0.53 & 23.08 & 0.04 & 0.27 \\
6 & 344.37478 & +41.428685 & 26.29 & 0.39 & 0.53 & 25.23 & 0.25 & 0.27 \\
7 & 344.37479 & +41.410686 & 26.32 & 0.40 & 0.53 & 25.55 & 0.27 & 0.27 \\
8 & 344.37481 & +41.397515 & 22.83 & 0.02 & 0.54 & 20.52 & 0.01 & 0.28 \\
9 & 344.37486 & +41.430424 & 24.32 & 0.07 & 0.53 & 23.45 & 0.04 & 0.27 \\
10 & 344.37489 & +41.383481 & 25.37 & 0.26 & 0.54 & 25.27 & 0.31 & 0.28 \\
\hline
\end{tabular}

Note-This table is available in its entirety in a machine-readable form in the online journal. A small portion of the data is shown as an example of the form and content of the table. 
Table 2. Properties of Lac I

\begin{tabular}{lr}
\hline \hline \multicolumn{1}{c}{ Property } & \multicolumn{1}{c}{ Value } \\
\hline & \\
Right Ascension $(\alpha)$ & $22 \mathrm{~h} 58 \mathrm{~m} 13.26 \mathrm{~s} \pm 0.19 \mathrm{~s}$ \\
Declination $(\delta)$ & $41^{\circ} 17^{\prime} 53.5^{\prime \prime} \pm 2.1^{\prime \prime}$ \\
$M_{V}(\mathrm{mag})$ & $-11.4 \pm 0.3$ \\
$M_{g}(\mathrm{mag})$ & $-11.3 \pm 0.2$ \\
$(g-i)_{o}(\mathrm{mag})$ & $0.7 \pm 0.3$ \\
$(m-M)_{0}(\mathrm{mag})$ & $24.44 \pm 0.11$ \\
$D(\mathrm{kpc})$ & $773 \pm 40$ \\
$D_{\mathrm{M} 31}(\mathrm{kpc})$ & $264 \pm 6$ \\
$\mu_{V, 0}\left(\mathrm{mag} / \mathrm{arcsec}{ }^{2}\right)$ & $24.8 \pm 0.3$ \\
$r_{h}(\mathrm{arcmin})$ & $3.24 \pm 0.21$ \\
$r_{h}(\mathrm{pc})$ & $728 \pm 47$ \\
Ellipticity $(\epsilon)$ & $0.44 \pm 0.03$ \\
Position angle $(\theta)^{\circ}$ East of North $)$ & $116.1 \pm 5.4$ \\
$M_{H I} / L_{V}\left(M_{\odot} / L_{\odot}\right)$ & $<0.06$ \\
{$[\text { Fe/H }]_{\text {med }}(\mathrm{dex})$} & $-1.68 \pm 0.03$ \\
\hline
\end{tabular}

